\title{
Applications of Forward Mortality Factor Models in Life Insurance Practice*
}

\author{
Nan Zhu and Daniel Bauer \\ Department of Risk Management and Insurance, Georgia State University, 35 Broad Street, $11^{\text {th }}$ Floor, \\ Atlanta, GA 30303, U.S. \\ E-mails: nzhu1@student.gsu.edu; dbauer@gsu.edu
}

Two of the most important challenges for the application of stochastic mortality models in life insurance practice are their complexity and their apparent incompatibility with classical life contingencies theory, which provides the backbone of insurers' Electronic Data Processing systems. Forward Mortality Factor Models comprise one model class that overcomes these challenges. Relying on a simple model version that originates from a semiparametric estimation based on British population mortality data, this paper demonstrates the merits of this model class by discussing several practically important example applications. In particular, we calculate the Economic Capital for a stylised life insurer, we present a closed-form solution for the value of a Guaranteed Annuity Option, and we derive the fair option fee for a Guaranteed Minimum Income Benefit within a Variable Annuity contract. Our numerical results illustrate the economic significance of systematic mortality risk.

The Geneva Papers (2011) 36, 567-594. doi:10.1057/gpp.2011.24

Keywords: Stochastic mortality models; forward mortality factor models; economic capital; Guaranteed Annuity Options; Guaranteed Minimum Income Benefits

\section{Introduction}

Two of the most important challenges for the application of stochastic mortality models in life insurance practice are the apparent incompatibility of most stochastic methods with classical life contingencies theory, which presents the backbone of insurers' Electronic Data Processing (EDP) systems, and the complexity of many of the proposed approaches. These obstacles have not only led to an increasing discrepancy between life insurance research and actuarial practice and education in some parts of the world, but the reluctance of practitioners to rely on stochastic mortality models may also be a primary reason for the sluggish development of the mortality-linked capital market. Specifically, stochastic methods are necessary to assess a company's capital relief when hedging part of its mortality risk exposure, which should be one of the key drivers of the demand for mortality-linked securities.

\footnotetext{
* This paper is an updated and extended version of the application part of the paper "Gaussian Forward Mortality Factor Models: Specification, Calibration, and Application" presented at the Sixth International Longevity Risk and Capital Markets Solutions Conference. Parts of it are taken from the second author's doctoral dissertation (cf. Bauer, 2009).
} 
One model class that overcomes these problems are so-called forward mortality models, which infer dynamics on the entire age/term-structure of mortality. As already pointed out by Milevsky and Promislow, ${ }^{1}$ the "traditional rates used by actuaries" really are forward rates so that the forward approach can be viewed as the natural extension of traditional actuarial theory. In particular, the actuarial present values for traditional insurance products such as term-life insurance, endowment insurance or life annuity contracts are of the same form as in classical actuarial theory, where the "survival probabilities" now are to be interpreted as expected values of realised survival probabilities. ${ }^{2}$ Hence, the inclusion of such models in the operations of a life insurer or a pension fund will not require alterations in the management of traditional product lines, but nonetheless present a coherent way to take mortality risk into account when necessary. Examples of such situations include the calculation of economic capital based on internal models or the pricing and risk management of mortality-linked guarantees in life insurance or pension products.

However, only few forward mortality models have been proposed so far, and most authors have relied on "qualitative" insights and/or modelling convenience for determining suitable specifications. ${ }^{3}$ Moreover, some of the presented models entail a high degree of complexity, which may lead to problems in their calibration. ${ }^{4}$

In a companion paper, ${ }^{5}$ we present an alternative, data-driven approach by relying on forward mortality factor models with Normal-distributed transition factors, the (necessary) explicit functional form for which has been identified by Bauer et al. ${ }^{6}$ More specifically, we use principal component analyses of time series of mortality forecasts generated based on rolling windows of annual mortality data to derive a suitable number of stochastic factors and their functional forms. The resulting specifications are then (re)calibrated based on maximum likelihood estimation (MLE) (the main ideas and relevant results are summarised in the next section and the Appendix). In this paper, we demonstrate the technical advantages of this model class by discussing and implementing several important example applications. Furthermore, our numerical results based on a simple model version calibrated to British population mortality data illustrate the economic significance of systematic mortality risk.

The first application concerns the calculation of economic capital for life insurance companies. After providing a framework for this problem similar to that from Bauer et al., ${ }^{7}$ we explicitly demonstrate how to derive the economic capital for a stylised life insurance company offering traditional life insurance products in our setting. Our implementation highlights the tractability of forward mortality factor models as well as the important advantage of this model class in that it avoids the necessity of nested simulations. ${ }^{8}$ Furthermore, our numerical results display that in addition to financial

\footnotetext{
${ }^{1}$ Milevsky and Promislow (2001).

${ }^{2}$ Cf. Bauer et al. (2010a).

${ }^{3}$ Cf. Bauer et al. (2008a), Dawson et al. (2010), or Schrager (2006).

${ }^{4}$ See e.g. Bauer et al. (2008a).

${ }^{5}$ Zhu and Bauer (2011).

${ }^{6}$ Bauer et al. (2010a).

${ }^{7}$ Bauer et al. (2010b).

${ }^{8}$ For an overview on Monte Carlo methods in financial modelling, see for example Glasserman (2004).
} 
risk, (systematic) mortality risk has a considerable impact on the results and thus plays an important role for a life insurance company's solvency.

In the second application, we discuss the valuation of different mortality-contingent embedded options within life insurance contracts. Specifically, we derive a closed-form valuation formula for simple Guaranteed Annuity Options (GAOs) within traditional endowment policies in the considered forward mortality model framework. Moreover, we demonstrate how to derive the fair option fee for Guaranteed Minimum Income Benefits (GMIBs) within Variable Annuity contracts based on Monte Carlo simulations; here, akin to the first application, forward mortality models bear the profound advantage that no nested simulations are necessary. Our numerical results again emphasise the economic significance of systematic mortality risk.

The remainder of the paper is organised as follows: The next section provides a short summary of the considered mortality modelling framework from Zhu and Bauer $^{5}$ and introduces the model underlying our implementations. The two subsequent sections present our applications to economic capital modelling and the valuation of annuitisation options, respectively. Finally, the last section concludes.

\section{Forward mortality factor models}

In a best estimate generation life table at time $t \geqslant 0$, forward survival probabilities ${ }_{\tau} p_{x}(t, t+\tau)$ are listed for a (large) collection of ages $x \geqslant 0$ and terms $\tau \geqslant 0$, where ${ }_{\tau} p_{x}(t, T+\tau)$ is the $\mathcal{F}_{t}$-measurable random variable satisfying ${ }^{9}$

$$
\begin{gathered}
{ }_{\tau} p_{x}(t, T+\tau) \mathbf{1}_{\left\{\Upsilon_{x-T}>T\right\}}=\mathbb{E}^{\mathbb{P}}\left[\mathbf{1}_{\left\{\Upsilon_{x-T}>T+\tau\right\}} \mid \mathcal{F}_{t} \vee\left\{\Upsilon_{x-T}>T\right\}\right], \\
0 \leqslant T \leqslant t \leqslant T+\tau,
\end{gathered}
$$

and $\Upsilon_{x_{0}}$ is the (random) time of death or future lifetime of an $x_{0}$-year old at time zero. Hence, ${ }_{\tau} p_{x}(t, T+\tau)$ denotes the - at time $t$-expected $\tau$-year survival probability for an $x$-year old at time $T$, whereas ${ }_{\tau} p_{x}(T+\tau, T+\tau)$ denotes the corresponding realised survival probability.

We introduce the so-called forward force of mortality

$$
\mu_{t}(\tau, x)=-\frac{\partial}{\partial \tau} \log \left\{\tau p_{x}(t, t+\tau)\right\}
$$

as a-from a modelling perspective - convenient representation of the forward survival probabilities constituting the generation life table at $t$, so that we have

$$
{ }_{\tau} p_{x}(t, t+\tau)=\exp \left\{-\int_{0}^{\tau} \mu_{t}(s, x) d s\right\} .
$$

\footnotetext{
${ }^{9}$ As usually in this context, underlying our considerations is a filtered probability space $\left(\Omega, \mathcal{H}, \mathbf{F}=\left(\mathcal{F}_{t}\right)_{t \geqslant 0}, \mathbb{P}\right)$. Here, the filtration $\mathbf{F}$ satisfies the usual conditions and models the information flow of aggregate population dynamics, whereas the sigma algebra $\mathcal{H}$ also contains information about individual deaths within the population. We refer to Bauer et al. (2010a) for technical details.
} 
A (forward) mortality model now specifies the evolution of the generation life tables

$$
\left({ }_{\tau} p_{x}(t, t+\tau), x, \tau \geqslant 0\right)_{t \geqslant 0},
$$

or equivalently $\left(\mu_{t}(\tau, x), x, \tau \geqslant 0\right)_{t \geqslant 0}$, over time, which can be formulated as a stochastic (differential) equation of the form ${ }^{2}$

$$
d \mu_{t}=\left(A \mu_{t}+\alpha_{t}\right) d t+\sigma_{t} d W_{t}, \mu_{0}(\cdot, \cdot)>0,
$$

where $\alpha_{t}$ and $\sigma_{t}$ are adequate, function-valued stochastic processes, $A=(\partial / \partial \tau)-(\partial / \partial x)$, and $\left(W_{t}\right)$ is a $d$-dimensional Brownian motion. Furthermore, if the dynamics (1) are formulated under $\mathbb{P}$, we have the drift condition ${ }^{10}$

$$
\alpha_{t}(\tau, x)=\sigma_{t}(\tau, x) \times \int_{0}^{\tau} \sigma_{t}^{\prime}(s, x) d s .
$$

Hence, to specify a model, it is sufficient to specify a suitable volatility structure $\left(\sigma_{t}(\tau, x), x, \tau \geqslant 0\right)_{t \geqslant 0}$ in $\mathbb{R}^{d}$.

In Zhu and Bauer, ${ }^{5}$ we consider time-homogenous models of this type for which transitions can be realised by Normal-distributed random vectors, that is models of the form

$$
\mu_{t}(\tau, x)=G\left(\tau, x, Z_{t}\right)
$$

for some Normal-distributed random vector $Z_{t}$, which have been studied in detail in Bauer et al. ${ }^{6}$ In particular, the authors show that the volatility structure must necessarily be of the form

$$
\sigma(\tau, x)=C(x+\tau) \times \exp \{M \tau\} \times N,
$$

where $N \in \mathbb{R}^{m \times d}, M \in \mathbb{R}^{m \times m}$, and $C^{\prime} \in C^{1}\left([0, \infty), \mathbb{R}^{m}\right)$, so that

$$
\begin{aligned}
\mu_{t}(\tau, x)= & \mu_{0}(\tau+t, x-t)+\int_{0}^{t} \alpha(\tau+t-s, x-t+s) d s \\
& +C(x+\tau) \exp \{M \tau\} \underbrace{\int_{0}^{t} \exp \{M(t-s)\} N d W_{s}}_{=Z_{t}} .
\end{aligned}
$$

While the confinement to Normal distributions implies the theoretical shortcoming that realisations of survival probabilities - with a small probability-may exceed unity, it allows us to derive adequate specifications using a principal component analysis. Furthermore, as also shown in Zhu and Bauer, ${ }^{5}$ it is easy to devise a maximum likelihood approach for the (re)calibration of the resulting specifications

${ }^{10}$ Cf. Cairns et al. (2006), Bauer et al. (2010a). 
that explicitly takes the drift condition (2) into account. To cast suitable specifications, we rely on mortality forecasts generated using different forecasting methods and rolling windows of annual mortality data for various countries. Our analyses show that the first one to two principal components capture the great majority of all the variation in the data, and the corresponding error terms exhibit systematic shapes that can be captured by few parameters.

In this paper, we also limit our focus to these type of models satisfying (3) since suitable specifications are immediately available from Zhu and Bauer, ${ }^{5}$ but also due to important advantages in applications that will be illustrated throughout this text. For our implementations, we adopt a parsimonious (one-factor) model version, where the underlying mortality forecasts were generated using the Lee and Carter ${ }^{11}$ methodology ${ }^{12}$ based on male mortality data from England and Wales for the years 1947-2006 as available from the Human Mortality Database. ${ }^{13}$ More precisely, we use observations for ages between 20 and 95 years to compile 31 consecutive generation life tables (1977-2007) each relying on the mortality experience of the previous 30 years with the Lee-Carter parameters $\left(\left\{\alpha_{x}\right\},\left\{\beta_{x}\right\}\right.$ and $\left.\left\{\kappa_{t}\right\}\right)$ calibrated independently and the random-walk drift for $\left(\kappa_{t}\right)$ re-estimated for each 30 -year window. Hence, the first table uses mortality data from 1947-1976, the second one uses 1948-1977, and so forth. As described in Zhu and Bauer, ${ }^{5}$ the resulting time series of tables can then be used to derive suitable specifications based on a principle component analysis. The results are displayed in Figure 1; more details on the underlying procedure are provided in the Appendix.

Figure 1(a) shows the volatility $\sigma$ associated with the first principal component as a function of $\tau$ and $x$, which explains more than 91 per cent of all the variation in the data. The (non-parametric) least-squares fit of $C$ is shown in Figure 1(b). The shape can be captured well by the specification ${ }^{14}$

$$
\begin{aligned}
\sigma(\tau, x)= & k \frac{\exp \{c(x+\tau)+d\}}{1+\exp \{c(x+\tau)+d\}}(a+\tau) e^{-b \tau}=\underbrace{\left(\begin{array}{ll}
0 & \left.k \frac{\exp \{c(x+\tau)+d\}}{1+\exp \{c(x+\tau)+d\}}\right)
\end{array}\right.}_{=C(x+\tau)} \\
& \times \underbrace{\exp \left\{\left(\begin{array}{cc}
-2 b & -b^{2} \\
1 & 0
\end{array}\right) \times \tau\right\}}_{=\exp \{M \tau\}} \times \underbrace{\left(\begin{array}{c}
1-a b \\
a
\end{array}\right)}_{=N} .
\end{aligned}
$$

The parameters are subsequently (re)calibrated based on an MLE method (see also the Appendix for more details), with the resulting estimates displayed in Table 1.

${ }^{11}$ Lee and Carter (1992).

${ }^{12}$ More specifically, $\left\{\alpha_{x}\right\}$ and $\left\{\beta_{x}\right\}$ in the Lee-Carter model are calibrated via the weighted least-squares algorithm, and $\left\{\kappa_{t}\right\}$ is further adjusted by fitting a Poisson regression model (cf. Booth et al., 2002).

${ }^{13}$ Human Mortality Database. University of California, Berkeley (U.S.), and Max Planck Institute for Demographic Research (Germany). Available at www.mortality.org or www.humanmortality.de.

${ }^{14}$ See also Björk and Gombani (1999). 

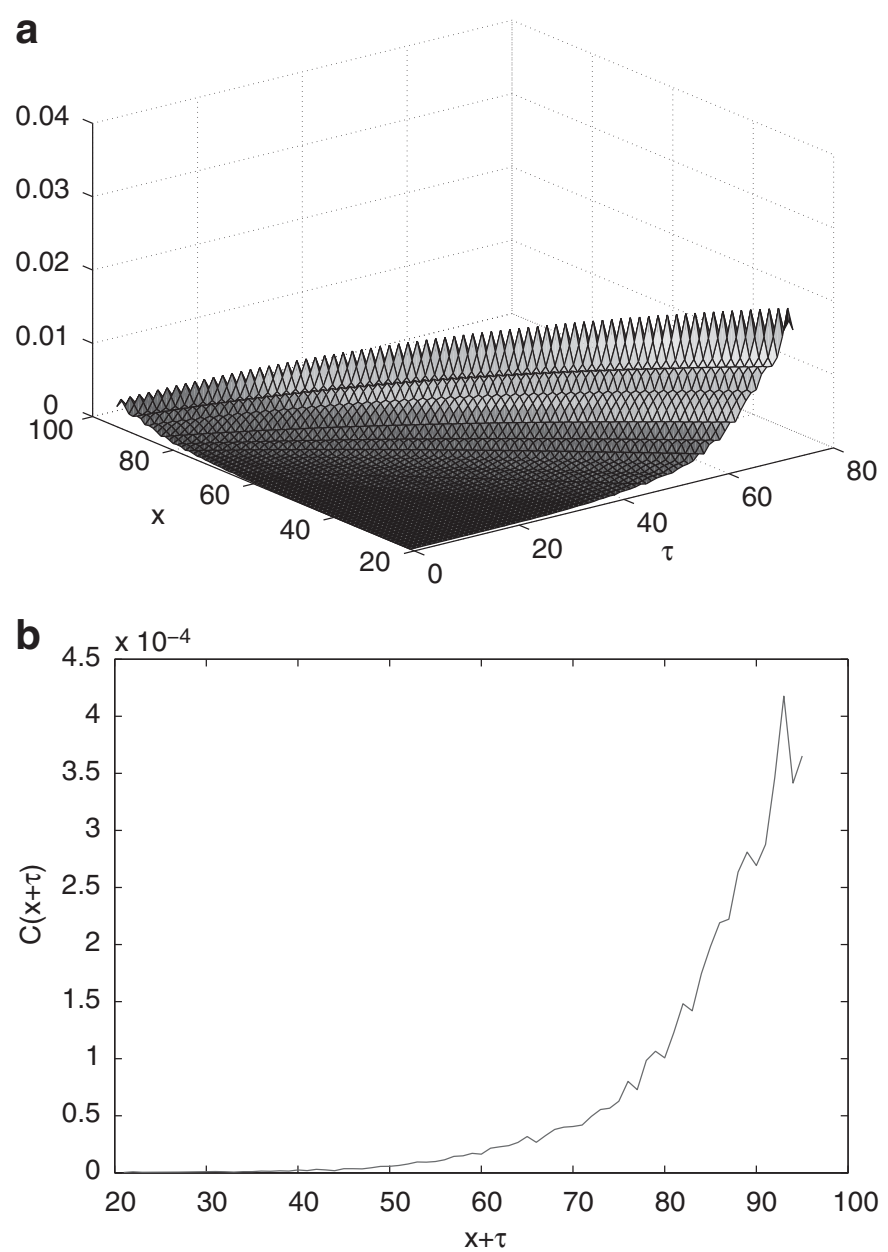

Figure 1. Principal component analysis, estimation and projection. (a) First principal component- sample data; (b) non-parametric fit of $C(x+\tau)$; (c) first principal component - projection; and (d) MLE estimate of $C(x+\tau)$.

Figure 1(c) shows the projection of the first principal component based on the MLE, while Figure 1(d) depicts $C(x+\tau)$ under the logistic-Gompertz functional form assumption after the MLE. The differences between Figures 1(a) and 1(c) arise since the MLE method explicitly takes the drift condition (2) into account.

\section{Application I: Economic capital in internal models}

As indicated above, one potential reason for the sluggish development of the mortality-linked capital market may be the struggle of insurers with the assessment of their capital relief when hedging part of their mortality risk exposure. In this section, 

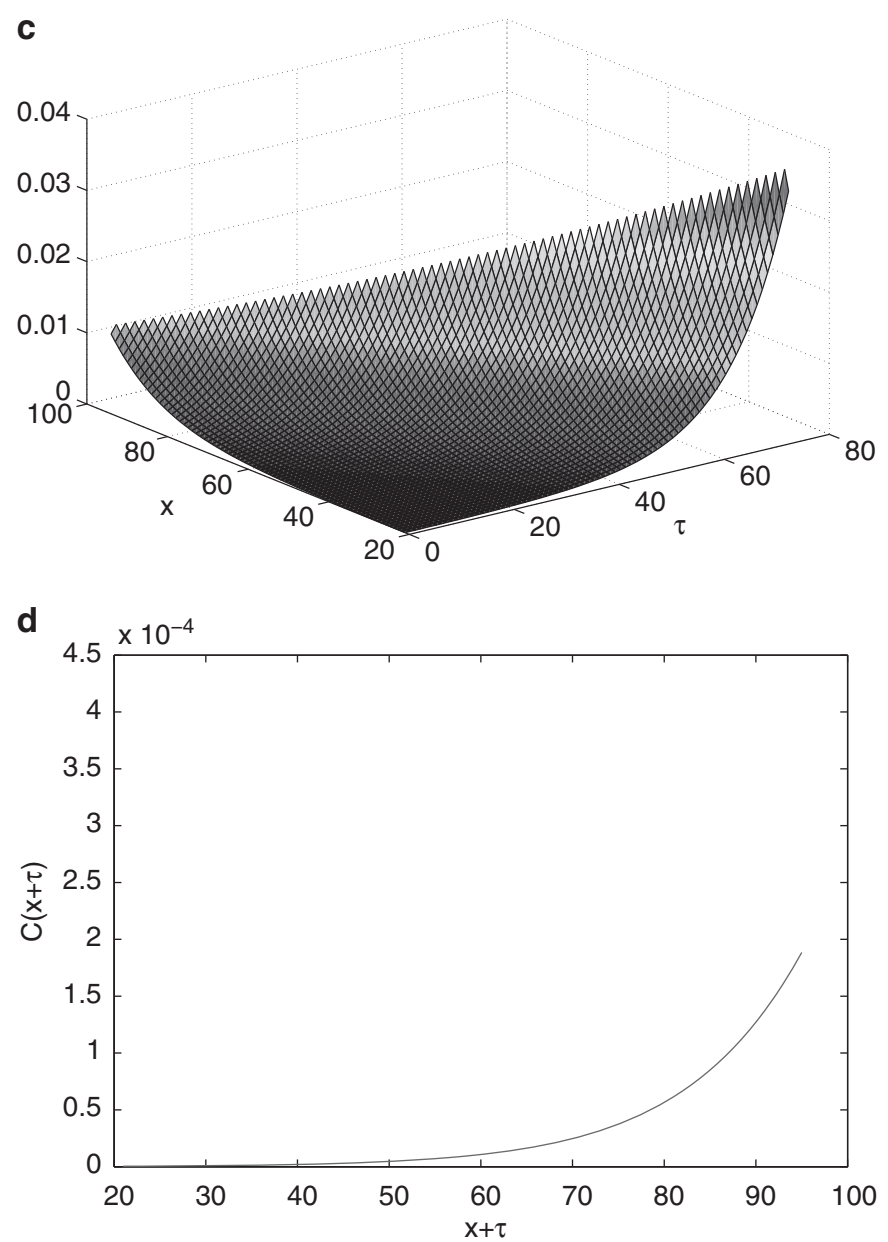

Figure 1. Continued

Table 1 Calibrated parameters from the MLE method

\begin{tabular}{lcccr}
\hline Parameters & & & \\
\hline$k$ & $c$ & $d$ & $a$ & $b$ \\
\hline 0.0025 & 0.0840 & -10.4692 & 62.9958 & -0.0052 \\
\hline
\end{tabular}

we demonstrate that due to their compatibility with classical actuarial methods and their tractability, forward mortality factor models present a pertinent and simple way for incorporating mortality risk into insurers' economic capital calculations. ${ }^{15}$

${ }^{15}$ See Börger (2010) and Stevens et al. (2010) for alternative approaches. 
We first introduce a mathematical framework similar to that from Bauer et al. ${ }^{7}$ for determining the economic capital in a one-year mark-to-market approach as required by the dawning Solvency II regulation. Subsequently, to describe in detail the merits of forward mortality factor models in this context but also to analyse the influence of systematic mortality risk on economic capital in a reasonably realistic setting, we carry out example calculations for a stylised insurance company offering traditional life insurance products.

\section{Model framework}

Assume the uncertainty with respect to a life insurer's future profits arises from the uncertain development of a number of financial/economic and demographic factors, which are modelled with the help of the $d_{Y}$-dimensional, sufficiently regular Markov process $Y=\left(Y_{t}\right)_{t \geqslant 0}=\left(Y_{t}^{(1)}, \ldots, Y_{t}^{\left(d_{Y}\right)}\right)_{t \geqslant 0}^{\prime}$, the so-called state process. More specifically, we assume that the prices of all risky assets in the market-including zero coupon bonds - can be expressed in terms of $Y_{t}$, and that there exists a locally risk-free asset (bank account) $B=\left(B_{t}\right)_{t \geqslant 0}$ with $B_{t}=\exp \left\{\int_{0}^{t} r_{s} d s\right\}$, where $r_{t}=r\left(Y_{t}\right)$ is the instantaneous risk-free rate at time $t$. Similarly, we assume that survival probabilities as denoted in the relevant life table at time $t$ can be formulated in terms of $Y_{t}$, that is ${ }_{\tau} p_{x}(t, t+\tau)={ }_{\tau} p_{x}\left(Y_{t}\right)$. In this market environment, we take for granted the existence of a risk-neutral probability measure $\mathbb{Q}$ equivalent to $\mathbb{P}$ under which payment streams can be valued via their expected discounted values with respect to the numéraire $B .^{16}$

On the basis of this economic/demographic environment, we assume that there exists a cash flow projection model, that is there exist functionals $f_{1}, \ldots, f_{T}$ that derive the cash flows at time $t$ from the state process up to time $t$, where $T$ is the time horizon. For instance, within the direct method for determining insurance liabilities, ${ }^{17}$ the random variable $X_{t}=f_{t}\left(Y_{s}, s \in[0, t]\right)$ corresponds to the benefits paid minus the premiums earned at time $t, t=1, \ldots, T$, and the value of the liabilities can be determined via

$$
V_{0}=\mathbb{E}^{\mathbb{Q}}\left[\sum_{k=0}^{T} \frac{1}{B_{k}} X_{k}\right] .
$$

Thus, the Available Capital (AC) at time zero can be derived from $V_{0}$ and the value of assets $A_{0}=A\left(Y_{0}\right)$ as

$$
A C_{0}=A_{0}-V_{0}
$$

However, within the one-year mark-to-market approach for calculating economic capital, it is not sufficient to determine the AC at time zero, but it is also necessary to

\footnotetext{
${ }^{16}$ According to the Fundamental Theorem of Asset Pricing, this assumption is essentially equivalent with the absence of arbitrage in the market.

${ }^{17}$ Cf. Girard (2002).
} 
assess $\mathrm{AC}$ at time $1, A C_{1}=A_{1}-V_{1}$, where

$$
V_{1}=X_{1}+B_{1} \mathbb{E}^{\mathbb{Q}}\left[\sum_{k=2}^{T} \frac{1}{B_{k}} X_{k} \mid Y_{s}, 0 \leqslant s \leqslant 1\right] .
$$

More specifically, the 1 -year loss is defined as the $\mathcal{F}_{1}$-measurable random variable

$$
L=A C_{0}-p(0,1) A C_{1},
$$

where $p(t, \tau)$ denotes the time $t$ price of a zero coupon bond with maturity $t+\tau$. The economic capital is then defined with the help of a monetary risk measure $\rho$ : $L^{2}(\Omega, \mathcal{F}, \mathbb{P}) \rightarrow \mathbb{R}$ as $\rho(L) .{ }^{18}$ For instance, if the economic capital (EC) is defined based on the Value-at-Risk (VaR) such as the Solvency Capital Requirement (SCR) within the Solvency II framework, we have

$$
\mathrm{EC}=\mathrm{SCR}=\operatorname{VaR}_{\alpha}(L)=\arg \min _{x}\{\mathbb{P}(L>x) \leqslant 1-\alpha\},
$$

where $\alpha$ is a given threshold (99.5 per cent within Solvency II). If economic capital is defined based on the Conditional Tail Expectation (CTE), on the other hand, we obtain

$$
\mathrm{EC}=\mathrm{CTE}_{\alpha}=\mathbb{E}\left[L \mid L \geqslant \operatorname{VaR}_{\alpha}(L)\right]
$$

\section{A stylised life insurance company}

Consider now a (stylised) newly founded life insurance company only selling traditional life insurance products to male individuals, who form a representative sample of the England and Wales general male population. More specifically, let us assume that the company's portfolio of policies consists of $n_{x, i}^{\text {term }} i$-year term-life policies for individuals aged $x$ with face value $B_{\text {term }}, n_{x, i}^{\text {end }} i$-year endowment policies for individuals aged $x$ with face value $B_{\text {end }}$, and $n_{x}^{\text {ann }}$ single premium life annuities with an annual benefit of $B_{\text {ann }}$ paid in arrears, $x \in \mathcal{X}, i \in \mathcal{I}$. Furthermore, we assume that the (for term and endowment policies annual) benefit premium is calculated by the Equivalence Principle disregarding profits as well as expenses, and using the concurrent yield curve and the concurrent best-estimate generation table. Here and for the remainder of the text, we implicitly assume that the insurer is risk-neutral with respect to mortality risk, that is the valuation measure $\mathbb{Q}$ is the product measure of the risk-neutral measure for financial and the physical measure for (independent) biometric events. This is without much loss of generality for the deterministic volatility forward mortality models satisfying (3) since, under the assumption of a deterministic market price of systematic mortality risk, a risk-adjusted generation table can be derived from the best estimate generation table via a deterministic transformation. ${ }^{19}$ This is another important advantage of the considered mortality model class in the present context.

\footnotetext{
${ }^{18}$ See for example Artzner et al. (1999).

${ }^{19}$ See Bauer et al. (2010a) and Bauer et al. (2010c) for details.
} 
Hence, the insurer's $\mathrm{AC}$ at time zero $A C_{0}$ amounts to its equity capital $E$. For the $\mathrm{AC}$ at time 1 , on the other hand, we have $A C_{1}=A_{1}-V_{1}$, where

$$
\begin{aligned}
& A_{1}=\left(E+B_{\text {ann }} \sum_{x \in \mathcal{X}} a_{x}(0) n_{x}^{\mathrm{ann}}+B_{\mathrm{term}} \sum_{x \in \mathcal{X}, i \in \mathcal{I}} \frac{A_{x: \bar{i}}(0)}{\ddot{a}_{x: \bar{i} \mid}(0)} n_{x, i}^{\mathrm{term}}\right. \\
& \left.+B_{\text {end }} \sum_{x \in \mathcal{X}, i \in \mathcal{I}} \frac{A_{x: \bar{i} \mid}(0)}{\ddot{a}_{x: \vec{i} \mid}(0)} n_{x, i}^{\text {end }}\right) \times R_{1}, \\
& V_{1}=B_{\mathrm{ann}} \sum_{x \in \mathcal{X}} \ddot{a}_{x+1}(1)\left(n_{x}^{\mathrm{ann}}-\mathcal{D}_{x}^{\mathrm{ann}}(0,1)\right)+B_{\mathrm{term}} \sum_{x \in \mathcal{X}, i \in \mathcal{I}} \mathcal{D}_{x, i}^{\mathrm{term}}(0,1) \\
& +B_{\text {end }} \sum_{x \in \mathcal{X}, i \in \mathcal{I}} \mathcal{D}_{x, i}^{\text {end }}(0,1) \\
& +B_{\text {term }} \sum_{x \in \mathcal{X}, i \in \mathcal{I}}\left[A_{x+1: \overline{i-1} \mid}(1)-\frac{A_{x: i \mid}(0)}{\ddot{a}_{x: \bar{i} \mid}(0)} \ddot{a}_{x+1: \overline{i-1} \mid}(1)\right] \\
& \times\left(n_{x, i}^{\mathrm{term}}-\mathcal{D}_{x, i}^{\mathrm{term}}(0,1)\right) \\
& +B_{\text {end }} \sum_{x \in \mathcal{X}, i \in \mathcal{I}}\left[A_{x+1: \overline{i-1} \mid}(1)-\frac{A_{x: \bar{i} \mid}(0)}{\ddot{a}_{x: \bar{i} \mid}(0)} \ddot{a}_{x+1: \overline{i-1} \mid}(1)\right] \\
& \times\left(n_{x, i}^{\mathrm{end}}-\mathcal{D}_{x, i}^{\mathrm{end}}(0,1)\right) .
\end{aligned}
$$

Here, $\left(R_{1}-1\right)$ is the return on the insurer's asset portfolio, $\mathcal{D}_{x, i}^{\text {con }}(0,1)$ is the number of deaths in the cohort of $x$-year old insureds with policies of term $i$ and of type con $\in\{$ ann, term, end $\}$, and $\ddot{a}_{x}(t), A_{x: \bar{i}}(t)$, etc. are the values of the contracts corresponding to the actuarial symbols calculated at time $t$ based on the yield curve and the generation table at time $t$. For instance,

$$
\ddot{a}_{x}(t)=\sum_{k=0}^{\infty}{ }_{k} p_{x}(t, t+k) p(t, k) .
$$

The economic capital of this insurer can then be determined as

$$
E C=\rho\left(E-\left(A_{1}-V_{1}\right) p(0,1)\right),
$$

where $\rho(\cdot)$ is a monetary risk measure as described above.

\section{Implementation}

We assume that our U.K. insurer only invests in one, three, five and ten-year government bonds as well as an equity index $S=\left(S_{t}\right)_{t \geqslant 0}$ (FTSE) at predetermined 
Table 2 Estimated capital market parameters

\begin{tabular}{lccccccc}
\hline Parameters & \multicolumn{10}{l}{} \\
\hline$\mu$ & $\sigma_{A}$ & $\rho$ & $\kappa$ & $\gamma$ & $\sigma_{r}$ & $\lambda$ & $r_{0}(06 / 2008)$ \\
\hline 0.1005 & 0.1429 & -0.2502 & 0.0998 & 0.0509 & 0.0090 & -0.1441 & 0.0473 \\
\hline
\end{tabular}

proportions. For the evolution of these assets, we assume a generalised Black-Scholes model with stochastic interest rates (Vasicek model), that is, under $\mathbb{P}$

$$
\begin{aligned}
d S_{t} & =S_{t}\left(\mu d t+\rho \sigma_{A} d B_{t}^{(1)}+\sqrt{1-\rho^{2}} \sigma_{A} d B_{t}^{(2)}\right), S_{0}>0, \\
d r_{t} & =\kappa\left(\gamma-r_{t}\right) d t+\sigma_{r} d B_{t}^{(1)}, r_{0}>0,
\end{aligned}
$$

where $\mu, \sigma_{A}, \kappa, \gamma, \sigma_{r}>0, \rho \in[-1,1]$, and $\left(B_{t}^{(1)}\right)$ and $\left(B_{t}^{(2)}\right)$ are independent Brownian motions under $\mathbb{P}$ that are independent of $\left(W_{t}\right)$. Moreover, we assume that the market price of interest rate risk is constant and denote it by $\lambda$, that is we replace $\mu$ by $r_{t}$ and $\gamma$ by $\gamma-\left(\lambda \sigma_{r} / \kappa\right)$ for the dynamics under the risk-neutral measure $\mathbb{Q}$ (we assume $\lambda<\left(\kappa / \sigma_{r}\right) \gamma$ to ensure $r_{t}$ is mean-reverting under $\mathbb{Q}$ ).

We estimate the parameters based on U.K. data from June 1988 to June 2008 using a Kalman filter. More precisely, we use monthly data for the FTSE 100 total return index, ${ }^{20}$ treasury bills (three months), ${ }^{21}$ and government bonds with maturities of one year, three years, five years and ten years. ${ }^{22}$ The resulting parameter estimates are displayed in Table $2 .^{23}$

Hence, based on realisations of the asset process and the instantaneous risk-free rate at time $1, R_{1}$ can be determined as

$$
R_{1}=\omega_{1} \frac{S_{1}}{S_{0}}+\omega_{2} \frac{1}{p(0,1)}+\omega_{3} \frac{p(1,2)}{p(0,3)}+\omega_{4} \frac{p(1,4)}{p(0,5)}+\omega_{5} \frac{p(1,9)}{p(0,10)},
$$

in which $\omega_{i}, i=1, \ldots, 5$, are predetermined proportions invested in each asset (for our numerical analyses, we impose equal proportions, that is $\omega_{i}=20$ per cent, $i=1, \ldots, 5)$.

${ }^{20}$ Downloaded on 5 November 2011 from Bloomberg (code: TUKXG).

${ }^{21}$ Downloaded on 5 November 2011 from the Bank of England's website, www.bankofengland.co.uk/mfsd/ $i a d b /$.

${ }^{22}$ Downloaded on 5 November 2011 from the Bank of England's website, bankofengland.uk/statistics/ yieldcurve/archive.htm.

${ }^{23}$ Some of the parameters from our estimation procedure - particularly $\rho$ and $\lambda$-slightly deviate from values used in other studies, which may be due to idiosyncrasies of the considered time period. To ensure that our results are not specific to the considered parameters, we conducted detailed sensitivity analyses for all of our applications. Since the analyses do not reveal new insights, in order to keep the presentation concise, these results are not included in the paper. 
To generate realisations, $r_{1}$ and $S_{1}$ are simulated from a joint Normal distribution. More specifically, we have:

$$
\begin{gathered}
r_{1}=e^{-\kappa} r_{0}+\gamma\left(1-e^{-\kappa}\right)+\int_{0}^{1} \sigma_{r} e^{-\kappa(1-s)} d B_{s}^{(1)}, \\
S_{1}=S_{0} \times \exp \left\{\mu-\frac{\sigma_{A}^{2}}{2}+\rho \sigma_{A} B_{1}^{(1)}+\sqrt{1-\rho^{2}} \sigma_{A} B_{1}^{(2)}\right\},
\end{gathered}
$$

which can be conveniently used in Monte Carlo algorithms. ${ }^{24}$ Bond prices can then be calculated in a straight-forward manner due to their exponential-affine structure, that is we have $p(t, \tau)=\exp \left(-A(\tau) r_{t}+C(\tau)\right)$, where

$$
A(\tau)=\frac{1}{\kappa}\left(1-e^{-\kappa \tau}\right)
$$

and

$$
C(\tau)=\left(\gamma-\frac{\lambda \sigma_{r}}{\kappa}-\frac{\sigma_{r}^{2}}{2 \kappa^{2}}\right)\left(\frac{1-e^{-\kappa \tau}}{\kappa}-\tau\right)-\frac{\sigma_{r}^{2}\left(1-e^{-\kappa \tau}\right)^{2}}{4 \kappa^{3}} .
$$

With respect to mortality risk, two different approaches are considered. In the first approach, we assume that mortality rates evolve deterministically, that is we use the last generation life table from our estimation procedure outlined in the second section and ignore systematic mortality risk. ${ }^{25}$ The realised deaths $\left(\mathcal{D}_{x, i}^{\text {con }}, \quad x \in \mathcal{X}, i \in \mathcal{I}, \quad\right.$ con $\in\{$ ann, term, end $\}$ ) are then simulated from a Binomial distribution using the corresponding one-year mortality rates denoted in the life table.

In the second approach, systematic mortality risk is considered by relying on the model introduced in the second section. More specifically, from Eq. (4) we obtain

$$
\begin{aligned}
& { }_{\tau} p_{x}(1,1+\tau)=\frac{{ }^{\tau+1} p_{x-1}(0,1+\tau)}{1 p_{x-1}(0,1)} \\
& \quad \times \exp \left\{-\int_{0}^{1} \int_{0}^{\tau} \alpha(v+1-s, x-1+s) d v d s-\int_{0}^{\tau} C(x+v) e^{M v} d v \times Z_{1}\right\},
\end{aligned}
$$

\footnotetext{
${ }^{24}$ See for example Zaglauer and Bauer (2008).

${ }^{25}$ For simplicity, we use a maximal age of $\bar{\omega}=95$ throughout this paper since we only rely on mortality data up to the age of 95 in the estimation procedure. This is without much loss of generality since probabilities for ages beyond 95 could be, for example, easily determined by extrapolation (see Börger, 2010).
} 
in which with Eq. (2)

$$
\begin{aligned}
& \int_{0}^{1} \int_{0}^{\tau} \alpha(v+1-s, x-1+s) d v d s \\
& \quad=\int_{0}^{1}\left\{\frac{1}{2} \int_{1-s}^{\tau+1-s} \sigma(u, x-1+s) d u \int_{1-s}^{\tau+1-s} \sigma^{\prime}(u, x-1+s) d u\right. \\
& \left.\quad+\int_{1-s}^{\tau+1-s} \sigma(u, x-1+s) d u \int_{0}^{1-s} \sigma^{\prime}(u, x-1+s) d u\right\} d s .
\end{aligned}
$$

From Eq. (5), on the other hand,

$$
e^{M v}=\left(\begin{array}{cc}
\left(1-b_{1} v\right) e^{-b_{1} v} & -b_{1}^{2} v e^{-b_{1} v} \\
v e^{-b_{1} v} & \left(1+b_{1} v\right) e^{-b_{1} v}
\end{array}\right)
$$

so that

$$
C(x+v) e^{M v}=\left(\frac{k_{1} e^{c_{1}(x+v)+d_{1}}}{1+e^{c_{1}(x+v)+d_{1}}} v e^{-b_{1} v} \frac{k_{1} e^{c_{1}(x+v)+d_{1}}}{1+e^{c_{1}(x+v)+d_{1}}}\left(1+b_{1} v\right) e^{-b_{1} v}\right) .
$$

Therefore, for each $Z_{1}$ simulated according to (4), Eq. (6) immediately yields a generation life table $\left\{{ }_{\tau} p_{x}(1 ; 1+\tau), x, \tau \geqslant 0\right\}$ at time 1 .

However, it is important to notice that solely relying on the simulated life tables at time one and otherwise proceeding as in the deterministic mortality approach will lead to a slight bias. More precisely, to obtain estimates that - on average - correspond to the deterministic mortality case, we also need to consider the stochastic evolution of mortality within the simulation of the numbers of deaths $\mathcal{D}_{x, i}^{\text {con }}, x \in \mathcal{X}, i \in \mathcal{I}$, con $\in\{$ ann, term, end $\}$, which for each cohort now follow Binomial distributions with a random mortality probability ${ }_{1} q_{x}(1,1)=1-{ }_{1} p_{x}(1,1)$, where

$$
\begin{aligned}
{ }_{1} p_{x}(1,1) & =\exp \left\{-\int_{0}^{1} \mu_{s}(0, x+s) d s\right\} \\
& =\exp \left\{-\int_{0}^{1}\left(\mu_{0}(s, x)+\int_{0}^{s} \alpha(s-u, x+u) d u+C(x+s) Z_{s}\right) d s\right\} \\
& ={ }_{1} p_{x}(0,1) \times \exp \left\{-\int_{0}^{1} \int_{0}^{s} \alpha(s-u, x+u) d u d s-\int_{0}^{1} C(x+s) Z_{s} d s\right\} .
\end{aligned}
$$

Thus, it is not sufficient to sample $Z_{1}$ only as for the simulation of ${ }_{\tau} p_{x}(1,1+\tau)$ according to Eq. (6), but it is necessary to simulate the first year path 
$\left(Z_{s}\right)_{0 \leqslant s \leqslant 1}$. Here, we can rely on an exact simulation of the increments since for $t>u$, we have

$$
\begin{aligned}
Z_{t} & =\int_{0}^{t} e^{M(t-s)} N d W_{s} \\
& =e^{M t} \times\left(e^{-M u} e^{M u} \int_{0}^{u} e^{-M s} N d W_{s}+\int_{u}^{t} e^{-M s} N d W_{s}\right) \\
& \stackrel{d}{=} e^{M(t-u)} Z_{u}+\underbrace{\int_{0}^{t-u} e^{M(t-u-v)} N d W_{v}}_{=Y},
\end{aligned}
$$

where $Y$ is Normal-distributed with mean 0 and $\operatorname{Cov}(Y)=\int_{0}^{t-u} e^{M(t-u-v)}$ $N N^{\prime} e^{M(t-u-v)^{\prime}} d v$. Then, (7) can be immediately simulated by approximating the integral via its left sum, that is for large $n^{26}$

$$
\int_{0}^{1} C(x+s) Z_{s} d s \approx \frac{1}{n} \sum_{i=0}^{n-1} C\left(x+\frac{i}{n}\right) Z_{i / n} .
$$

The above illustrates the distinct advantages of forward mortality factor models in this context: On the one hand, since the actuarial present values at time 1 are given by the simulated generation life tables, it is possible to avoid a nested simulation structure as it would arise when relying on popular mortality models such as the Lee-Carter model. On the other hand, in contrast to the reliance on other forward mortality models when calculating economic capital, where the entire mortality surface needs to be simulated, ${ }^{27}$ here it is sufficient to simulate the finite-dimensional diffusion process $\left(Z_{s}\right)_{0 \leqslant s \leqslant 1}$, which can be carried out in a straightforward manner. Therefore, these models present a coherent and feasible method for incorporating stochastic mortality into internal economic capital models.

\section{Results}

We consider a stylised insurer with portfolio parameters as displayed in Table 3 , and we use 25,000 simulations of the loss at time 1 generated according to the algorithm outlined in the previous subsections to calculate the monetary risk measures of interest. Furthermore, in order to obtain estimates of simulation errors, we repeat the above procedure 100 times. The empirical cumulative distribution functions of the portfolio loss $L$ for both approaches, that is without and with the consideration of systematic mortality risk, are displayed in Figure 2. Table 4 shows the results of economic capital calculations based on VaR and CTE.

\footnotetext{
${ }^{26}$ We choose $n=100$ within our calculations.

${ }^{27}$ See for example Börger (2010).
} 
Table 3 Portfolio for the company, $E=2,000,000$

\begin{tabular}{lrrr}
\hline$x$ & $i$ & $n_{x, i}^{\text {term/end/ann }}$ & $B_{\text {term/end } / \text { ann }}$ \\
\hline Term life & & & 100,000 \\
30 & 20 & 250 & 100,000 \\
35 & 15 & 250 & 100,000 \\
40 & 10 & 250 & 100,000 \\
45 & 5 & 250 & \\
& & & 50,000 \\
Endowment & & 500 & 50,000 \\
40 & 15 & 500 & 50,000 \\
45 & 10 & 500 & 18,000 \\
50 & & & 18,000 \\
Annuities & & 250 & \\
60 & $(35)$ & 250 & \\
70 & $(25)$ & & \\
\hline
\end{tabular}

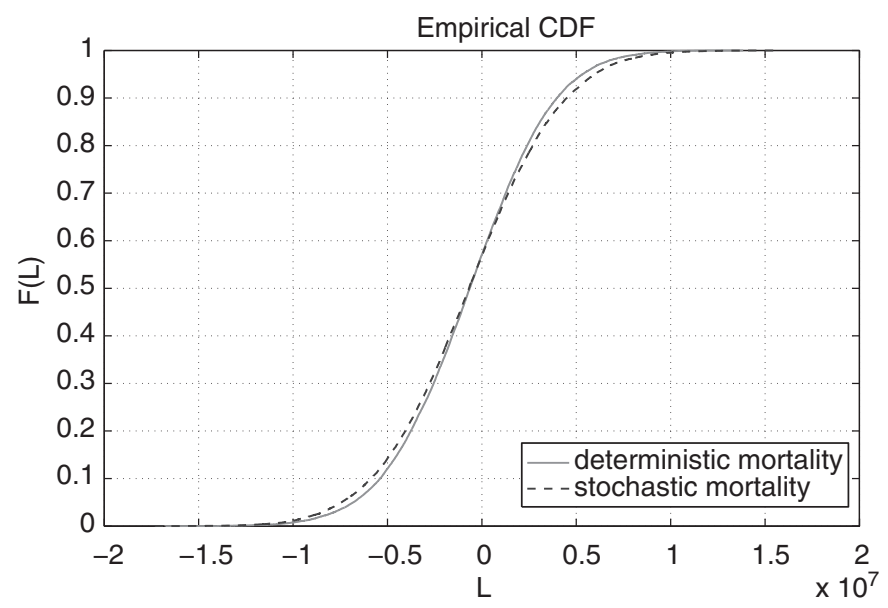

Figure 2. Empirical CDF of economic capital.

Table 4 Economic capital for the stylised company based on different risk measures

\begin{tabular}{lccrr}
\hline Confidence level $\alpha$ & VaR & Standard error & CTE & Standard error \\
\hline $\begin{array}{l}\text { Deterministic mortality } \\
90 \%\end{array}$ & $3,995,515$ & 39,596 & & 41,787 \\
$99 \%$ & $7,804,053$ & 93,629 & $5,712,494$ & 106,028 \\
& & & $9,047,340$ & \\
Stochastic mortality & & & & 45,018 \\
$90 \%$ & $4,494,682$ & 41,753 & $6,457,341$ & 118,670 \\
$99 \%$ & $8,844,264$ & 98,542 & $10,306,897$ & \\
\hline
\end{tabular}


Table 5 Calculations of $\mathrm{VaR}_{99 \%}$

\begin{tabular}{lrc}
\hline Evaluation technique & Stand-alone & Capital allocation \\
\hline Deterministic mortality & & 27,343 \\
$\quad$ Term life & 338,711 & $1,697,119$ \\
Endowment & $2,624,822$ & $6,079,590$ \\
Annuities & $5,961,906$ & $7,804,053$ \\
Total & $8,925,439$ & \\
& & $-120,123$ \\
Stochastic mortality & & $1,458,029$ \\
Term life & 473,474 & $7,506,357$ \\
Endowment & $2,652,375$ & $8,844,264$ \\
Annuities & $7,670,600$ & \\
Total & $10,796,450$ & \\
\hline
\end{tabular}

By comparing the results, we observe that the economic capital increases considerably when including systematic mortality risk: For the 90 per cent $(99$ per cent) threshold, the $\mathrm{VaR}$ increases by around 12 per cent (13 per cent) and the CTE increases by around 13 per cent (14 per cent). While the absolute levels have to be interpreted with care since we neither take expenses nor profits into account and since we do not consider a mortality risk premium associated with systematic mortality risk, our results demonstrate that mortality risk is an important risk factor and should be incorporated in risk-based capital calculations.

To investigate the effect of systematic mortality risk on the different types of insurance products, we calculate the 99 per cent VaR with respect to the three products separately under a stand-alone perspective ${ }^{28}$ and the capital allocated to each product line based on the covariance capital allocation technique for $\mathrm{VaR}$ described in Kalkbrener. ${ }^{29}$ The results are displayed in Table 5. While it is not surprising that the required capital of the total portfolio is smaller than the sum of the VaR of the individual product lines, we find that the different types of products are affected dissimilarly under systematic mortality risk. More precisely, the capital allocated to the term business within the portfolio is negative, implying a capital relief by exploiting natural hedging opportunities between the different lines. ${ }^{30}$ Nevertheless, the company's exposure to longevity risk still is considerable, and the capital relief by participating in the market for mortality-linked securities may be substantial.

\section{Application II: Valuation of annuitisation options}

As demonstrated in the previous section, traditional life insurance and pension products such as whole life, term life or endowment insurances as well as life annuities

\footnotetext{
${ }^{28}$ We assume the equity capital $E$ is distributed among different products according to their expected total premiums.

${ }^{29}$ Kalkbrener (2005)

${ }^{30}$ See also Cox and Lin (2007).
} 
may be evaluated directly using the "concurrent" (time $t$ ) mortality surface $\mu_{t}(\tau, x)$. For more complex life insurance and annuity contracts containing mortalitycontingent embedded options, however, the stochasticity has to be taken into account explicitly. Common types of longevity-contingent options are so-called GAOs within traditional or participating life insurance contracts and GMIBs within Variable Annuities. A GAO provides the policy-holder with the option to choose, at retirement, between a lump-sum payment or a life-long immediate annuity, which is calculated based on a guaranteed annuity rate. In contrast, a GMIB gives the insured the possibility to annuitise a guaranteed amount at a pre-specified rate. In this section, we provide a closed-form pricing formula for simple GAOs and an efficient numerical method for pricing GMIBs in our forward mortality modelling framework.

\section{Valuation of $G A O S$}

Several authors have studied the problem of evaluating GAOs without taking systematic mortality risk into account (see Boyle and Hardy, ${ }^{31}$ Pelsser, ${ }^{32}$ and references therein). In contrast, Milevsky and Promislow ${ }^{1}$ provide a discrete- and continuous-time pricing framework for simple annuitisation options that takes the stochasticity of mortality rates into account. Similarly, Ballotta and Haberman ${ }^{33}$ present a pricing approach for GAOs, which accounts for both interest rate and mortality risk. Since the solution for the price is not in closed form, they rely on Monte Carlo simulations for the derivation of their numerical results. Using affine processes for modelling the financial market as well as the (spot) mortality evolution, Biffis and Millossovich $^{34}$ also present a pricing framework for GAOs. Under some structural assumptions, they derive analytical solutions up to the computation of Fourier transforms and/or numerical integrals for various contract designs.

Following ideas by Cairns et al. ${ }^{35}$ in their annuity market model, we consider the valuation of simple GAOs in our forward mortality modelling framework. Specifically, we focus on contracts providing payoffs of the following form at time $T$ if the policyholder is alive:

$$
V_{T}^{\mathrm{GAO}}=\max \left\{1, g_{x_{0}, T}^{\mathrm{GAO}} \ddot{a}_{x_{0}+T}(T)\right\}
$$

Here, $g_{x_{0}, T}^{\mathrm{GAO}}$ is the guaranteed annuity rate under the GAO for annuitisation at time $T$ contracted at time 0 for a, then, $x_{0}$-year old. Thus, the contract may be interpreted as a $T$-year pure endowment policy with the additional option to annuitise at the fixed rate $g_{x_{0}, T}^{\mathrm{GAO}}$ at maturity. ${ }^{36}$

31 Boyle and Hardy (2003).

${ }^{32}$ Pelsser (2003).

${ }^{33}$ Ballotta and Haberman (2006).

${ }^{34}$ Biffis and Millossovich (2006).

${ }^{35}$ Cairns et al. (2006).

${ }^{36}$ Of course, this pure endowment part may easily be combined with a term insurance contract to obtain an endowment contract including a GAO, which are common contracts in many markets. 
We have

$$
V_{T}^{\mathrm{GAO}}=1+\underbrace{\ddot{a}_{x_{0}+T}(T) \max \left\{g_{x_{0}, T}^{\mathrm{GAO}}-\frac{1}{\ddot{a}_{x_{0}+T}(T)}, 0\right\}}_{=C_{T}^{\mathrm{GAO}}}
$$

and thus, ${ }^{37}$

$$
\begin{aligned}
V_{0}^{\mathrm{GAO}} & =\mathbb{E}^{\mathbb{Q}}\left[\mathbf{1}_{\left\{\Upsilon_{x_{0}}>T\right\}} e^{-\int_{0}^{T} r_{s} d s} V_{T}^{\mathrm{GAO}}\right] \\
& =p(0, T)_{T} p_{x_{0}}(0, T)+\underbrace{\mathbb{E}^{\mathbb{Q}}\left[e^{-\int_{0}^{T} \mu_{s}\left(0, x_{0}+s\right) d s} e^{-\int_{0}^{T} r_{s} d s} C_{T}^{\mathrm{GAO}}\right]}_{=C_{0}^{\mathrm{GAO}}} .
\end{aligned}
$$

Define $\left(X_{t}\right)_{0 \leqslant t \leqslant T}$ via

$$
X_{t}=\sum_{k=T}^{\infty} p(t, k-t) \underbrace{\mathbb{E}^{\mathbb{Q}}\left[e^{-\int_{0}^{k} \mu_{s}\left(0, x_{0}+s\right) d s} \mid \mathcal{F}_{t}\right]}_{={ }_{k} p_{x_{0}}(t, k)} .
$$

Then, for the value of the GAO,

$$
\begin{aligned}
C_{0}^{\mathrm{GAO}}=\mathbb{E}^{\mathbb{Q}} & {[\frac{e^{-\int_{0}^{T} \mu_{s}\left(0, x_{0}+s\right) d s}}{B_{T}} \overbrace{\sum_{k=T}^{\infty} p(T, k-T)_{k-T} p_{x_{0}+T}(T, k)}^{\ddot{a}_{x_{0}+T}(T)}} \\
& \left.\times\left(g_{x_{0}, T}^{\mathrm{GAO}}-\frac{e^{-\int_{0}^{T} \mu_{s}\left(0, x_{0}+s\right) d s}}{e^{-\int_{0}^{T} \mu_{s}\left(0, x_{0}+s\right) d s} \ddot{a}_{x_{0}+T}(T)}\right)\right] \\
= & \mathbb{E}^{+}\left[\frac{X_{T}}{B_{T}}\left(g_{x_{0}, T}^{\mathrm{GAO}}-\frac{e^{-\int_{0}^{T} \mu_{s}\left(0, x_{0}+s\right) d s}}{X_{T}}\right)^{+}\right]=X_{0} \mathbb{E}^{\mathbb{Q}_{X}}\left[\left(g_{x_{0}, T}^{\mathrm{GAO}}-\frac{T p_{x_{0}}(T, T)}{X_{T}}\right)^{+}\right],
\end{aligned}
$$

where $\mathbb{Q}_{X}$ is the equivalent martingale measure associated with the numéraire process $\left(X_{t}\right)$ (see e.g. Björk ${ }^{38}$ for details on the change of numéraire technique). In particular, the price process of a security with payoff ${ }_{T} p_{x_{0}}(T, T)$ at time $T$ discounted by $\left(X_{t}\right)$ will be a martingale under $\mathbb{Q}_{X}$. Hence, in order to evaluate the expectation in (8), we solely need to assess the volatility term of $\left(p(t, T-t){ }_{T} p_{x_{0}}(t, T) / X_{t}\right)$ and, thus, the volatility

${ }^{37}$ Again, akin to the previous section, for simplicity and without much loss of generality, we assume that the insurer is risk-neutral with respect to mortality risk.

38 Björk (1999). 
term of $\left(X_{t}\right)$. From Proposition 2.1 in Bauer et al. ${ }^{6}$ and Proposition 20.5 in Björk, ${ }^{38}$ we obtain that under $\mathbb{Q}^{39}$

$$
d X_{t}=\sum_{k=T}^{\infty} p(t, k-t){ }_{k} p_{x_{0}}(t, k)\left(S\left(t, k, x_{0}\right), v(t, k-t)\right) d\left(\begin{array}{c}
W_{t} \\
B_{t}^{(1)}
\end{array}\right),
$$

where $v(t, k)$ is the time $t$ volatility of a zero coupon bond maturing at time $t+k$ and

$$
S\left(t, k, x_{0}\right)=-\int_{0}^{k-t} \sigma\left(s, x_{0}+t\right) d s
$$

Therefore, the volatility of $\left(X_{t}\right)$ is given by

$$
\begin{aligned}
& \sum_{k=T}^{\infty} p(t, k-t)_{k} p_{x_{0}}(t, k)\left(S\left(t, k, x_{0}\right), v(t, k-t)\right) \\
& =X_{t} \sum_{k=T}^{\infty} \underbrace{\frac{p(t, k-t)_{k} p_{x_{0}}(t, k)}{\sum_{l=T}^{\infty} p(t, l-t){ }_{l} p_{x_{0}}(t, l)}}_{=w_{t}(k)}\left(S\left(t, k, x_{0}\right), v(t, k-t)\right) .
\end{aligned}
$$

By an application of Itô's Lemma, the volatility of $\left(p(t, T-t){ }_{T} p_{x_{0}}(t, T) / X_{t}\right)$ is then given by

$$
\begin{aligned}
& \left(\frac{p(t, T-t)_{T} p_{x_{0}}(t, T)}{X_{t}}\right)\left(\left(S\left(t, T, x_{0}\right), v(t, T-t)\right)-\sum_{k=T}^{\infty} w_{t}(k)\left(S\left(t, k, x_{0}\right), v(t, k-t)\right)\right) \\
= & \left(\frac{p(t, T-t)_{T} p_{x_{0}}(t, T)}{X_{t}}\right) \underbrace{\left(\sum_{k=T}^{\infty} w_{t}(k)\left(\left(S\left(t, T, x_{0}\right), v(t, T-t)\right)-\left(S\left(t, k, x_{0}\right), v(t, k-t)\right)\right)\right)}_{\gamma\left(t, T, x_{0}\right)} .
\end{aligned}
$$

If now $\gamma(., .,$.$) were deterministic, we would be able to derive an analytical expression$ for (8) via a Black-type formula (this is basically the idea of Cairns et al., ${ }^{35}$ who propose to directly model the forward annuity rate $\left.\left(p(t, T-t)_{T} p_{x_{0}}(t, T) / X_{t}\right)\right)$. However, in our framework, the weights $w_{t}(k)$ are in fact stochastic. Nevertheless, such an approach may be understood as an approximation, and $\operatorname{Pelsser}^{32}$ points out that for the deterministic mortality case, one may infer $\gamma\left(t, T, x_{0}\right)$ by "freezing" the

${ }^{39}$ If not noted otherwise, we adopt the capital market model introduced in the section "Application I: Economic capital in internal models". 
stochastic weights at their current values. This is similar to a common approximation in the popular LIBOR market models (see Theorem 3.2 in Brace et al. ${ }^{40}$ ). In particular, since $v(\cdot, \cdot)$ is deterministic, in our problem we may fix

$$
\gamma\left(t, T, x_{0}\right) \approx \sum_{k=T}^{\infty} w_{0}(k)\left(\left(S\left(t, T, x_{0}\right), v(t, T-t)\right)-\left(S\left(t, k, x_{0}\right), v(t, k-t)\right)\right),
$$

which then yields:

$$
C_{0}^{\mathrm{GAO}} \approx X_{0}\left(g_{x_{0}, T}^{\mathrm{GAO}} \Phi\left(-d_{2}^{\mathrm{GAO}}\right)-\frac{p(0, T)_{T} p_{x_{0}}(0, T)}{X_{0}} \Phi\left(-d_{1}^{\mathrm{GAO}}\right)\right)
$$

where

$$
\begin{aligned}
d_{1}^{\mathrm{GAO}} & =\frac{\log \left\{\frac{p(0, T)_{T} p_{x_{0}}(0, T)}{X_{0}{g_{x_{0}}, T}^{\mathrm{AOO}}}\right\}+\frac{1}{2} \sigma_{\mathrm{GAO}}^{2}}{\sigma_{\mathrm{GAO}}}, \\
d_{2}^{\mathrm{GAO}} & =d_{1}^{\mathrm{GAO}}-\sigma_{\mathrm{GAO}}, \\
\sigma_{\mathrm{GAO}}^{2} & =\int_{0}^{T}\left\|\gamma\left(u, T, x_{0}\right)\right\|^{2} d u,
\end{aligned}
$$

and $\Phi(\cdot)$ denotes the cumulative distribution function of the standard Normal distribution.

It is important to note that we restricted ourselves to constant payoffs. In particular, this means that the GAO considered here provides a constant annuity guarantee, and thus, in this special case, it coincides with a GMIB within a "non-variable" VAcontract as introduced above. In practice, GAOs are also often attached to unit-linked or participating policies, and GMIBs are usually granted within "truly variable" VA contracts; in these cases, the lump-sum payment will be stochastic, and hence, formula (9) does not apply. ${ }^{41}$ Thus, one may have to resort to numerical methods for the valuation.

\section{Valuation of GMIBs}

For the time zero value of a Variable Annuity contract including a GMIB with guaranteed annuity payment $g_{x_{0}, T}^{\mathrm{GMIB}}$ per invested unit for an $x_{0}$-year old insured when

${ }^{40}$ Brace et al. (1997).

${ }^{41}$ Ballotta and Haberman (2003) provide pricing formulas for GAOs within unit-linked policies for a deterministic mortality evolution by applying the ideas of Jamshidian (1989). However, their results cannot be easily carried over to the stochastic mortality environment unless interest rates and the mortality evolution are driven by the same one-dimensional Brownian motion $W$, which seems very unrealistic. 
annuitising at time $T$ and no death benefit guarantee, that is in the case of death only the current account value is paid out at the end of the year of death, we have:

$$
\begin{aligned}
V_{0}^{\mathrm{GMIB}}= & \mathbb{E}^{\mathbb{Q}}\left[\mathbf{1}_{\left\{\Upsilon_{x_{0}}>T\right\}} e^{-\int_{0}^{T} r_{s} d s} \max \left\{g_{x_{0}, T}^{\mathrm{GMIB}} A_{0} \ddot{a}_{x_{0}+T}(T), A_{T}\right\}\right] \\
& +\sum_{k=0}^{T-1} \mathbb{E}^{\mathbb{Q}}\left[\mathbf{1}_{\left\{\Upsilon_{x_{0}} \in[k, k+1)\right\}} e^{-\int_{0}^{k+1} r_{s} d s} A_{k+1}\right]=\mathbb{E}^{\mathbb{Q}}\left[e^{-\int_{0}^{T} r_{s}+\mu_{s}\left(0, x_{0}+s\right) d s}\right. \\
& \left.\max \left\{g_{x_{0}, T}^{\mathrm{GMIB}} A_{0} \sum_{k=T}^{\infty} p(T, k-T)_{k-T} p_{x_{0}+T}(T, k), A_{T}\right\}\right] \\
& +A_{0} \sum_{k=0}^{T-1}\left({ }_{k} p_{x_{0}}(0, k)-{ }_{k+1} p_{x_{0}}(0, k+1)\right) e^{-\phi(k+1)} .
\end{aligned}
$$

Here $\left(A_{t}\right)$ denotes the insured's account value at time $t$, which - in our generalised Black-Scholes framework - is assumed to evolve according to the stochastic differential equation ${ }^{42}$

$$
d A_{t}=A_{t}\left(\left(r_{t}-\phi\right) d t+\rho \sigma_{A} d \tilde{B}_{t}^{(1)}+\sqrt{1-\rho^{2}} \sigma_{A} d \tilde{B}_{t}^{(2)}\right), A_{0}>0
$$

where $\left(\tilde{B}_{t}^{(1)}\right)$ and $\left(\tilde{B}_{t}^{(2)}\right)$ are independent Brownian motions under $\mathbb{Q}$ independent of $\left(W_{t}\right)$, and $\phi$ denotes the continuously deducted option fee. Now, methods similar to those proposed in Bauer et al., ${ }^{43}$ where a deterministic evolution of mortality is assumed, can be employed to determine the value. Similarly as in the first application, Eq. (10) reconfirms the advantage of forward mortality models and illustrates why Cairns ${ }^{44}$ considers them to be ideal for pricing contracts with embedded options: Within other mortality models, $\ddot{a}_{x_{0}+T}(T)$ is typically not available, but each "simulation" will require a "further bundle of simulations from time $T$ to evaluate forward survival probabilities [i.e. a nested simulation structure]. In contrast, forward survival probabilities are a standard part of the output [of forward force models] at time $T$ ".

\section{Implementation and results}

Guaranteed annuity options

For GAOs, we consider the guaranteed annuity rate

$$
g_{x_{0}, T}^{\mathrm{GAO}}=\frac{p(0, T)_{T} p_{x_{0}}(0, T)}{{ }_{T \mid} \ddot{a}_{x_{0}}(0)},
$$

\footnotetext{
${ }^{42}$ Cf. Bauer et al. (2008b).

43 Bauer et al. (2008b).

${ }^{44}$ Cairns (2007).
} 
in which $T \ddot{a}_{x_{0}}(0)=\ddot{a}_{x_{0}}(0)-\ddot{a}_{x_{0}: T}(0)$ is the present value of a $T$-year deferred annuity at time zero. Note that this is exactly the rate policy-holders would obtain if they committed to annuitising, that is our GAO is "at the money". Therefore,

$$
\frac{p(0, T)_{T} p_{x_{0}}(0, T)}{X_{0} g_{x_{0}, T}^{\mathrm{GAO}}}=1
$$

and

so that

$$
d_{1}^{\mathrm{GAO}}=-d_{2}^{\mathrm{GAO}}=\frac{1}{2} \sigma_{\mathrm{GAO}}^{2},
$$

$$
\begin{aligned}
C_{0}^{\mathrm{GAO}} & \approx X_{0}\left(g_{x_{0}, T}^{\mathrm{GAO}} \Phi\left(\frac{1}{2} \sigma_{\mathrm{GAO}}^{2}\right)-g_{x_{0}, T}^{\mathrm{GAO}} \Phi\left(-\frac{1}{2} \sigma_{\mathrm{GAO}}^{2}\right)\right) \\
& =p(0, T)_{T} p_{x_{0}}(0, T)\left(2 \Phi\left(\frac{1}{2} \sigma_{\mathrm{GAO}}^{2}\right)-1\right) .
\end{aligned}
$$

Furthermore, notice that ${ }^{45}$

$$
v(t, \tau)=-\sigma_{r} \int_{0}^{\tau} e^{-\kappa s} d s .
$$

Hence, from Eqs. (11) and (12), we can calculate guaranteed annuity rates and prices of GAOs for three representative age/term combinations (note that for a deterministic evolution of mortality, our approach coincides with that from Pelsser ${ }^{32}$ ). The results for the case without and with considering systematic mortality risk are displayed in Table 6.

We find that systematic mortality risk has a significant impact on the valuation of simple GAOs. We also observe different patterns of $C_{0}^{\mathrm{GAO}}$ with respect to maturity $T$ in the two cases: While $C_{0}^{\mathrm{GAO}}$ is decreasing in $T$ in either situation, the marginal effect of $T$ on $C_{0}^{\mathrm{GAO}}$ is relatively higher when there is no mortality risk. This appears to be a consequence of the exponential decrement of $v(t, \tau)$ with respect to $\tau$ for the volatility of the zero coupon bond against an increasing pattern of $S\left(t, t+\tau, x_{0}\right)$. More precisely, for an increasing maturity, the first two terms of (12) decrease while the last term is increasing in volatility and, thus, in $T$. It now seems that under a deterministic evolution of mortality, the first effect is more pronounced while under stochastic mortality, the increasing pattern of $S\left(t, t+\tau, x_{0}\right)$ implies a stronger impact of the latter effect. ${ }^{46}$

Thus, aside from considerable quantitative effects, stochastic mortality also affects the qualitative patterns of GAO values.

${ }^{45}$ Cf. Pelsser (2003).

${ }^{46}$ It is necessary to note that of course our results are affected by our choice of the interest rate model. Specifically, the volatilities of long-term interest rates implied by a one-factor Vasicek model may be too small to accurately capture the volatility of an annuity. We accept this shortcoming since our focus is on mortality risk but note that our quantitative results have to be interpreted with care. 
Table 6 Valuation of GAOs

\begin{tabular}{|c|c|c|c|}
\hline$\left(x_{0}, T\right)$ & $g_{x_{0}, T}^{\mathrm{GAO}}$ & $V_{0}^{\mathrm{GAO}}$ & $C_{0}^{\mathrm{GAO}}$ \\
\hline \multicolumn{4}{|c|}{ Deterministic mortality } \\
\hline$(30,35)$ & 0.0803 & 0.1315 & 0.0050 \\
\hline$(40,25)$ & 0.0825 & 0.2366 & 0.0088 \\
\hline$(50,15)$ & 0.0850 & 0.4242 & 0.0152 \\
\hline \multicolumn{4}{|c|}{ Stochastic mortality } \\
\hline$(30,35)$ & 0.0803 & 0.1377 & 0.0111 \\
\hline$(40,25)$ & 0.0825 & 0.2435 & 0.0158 \\
\hline$(50,15)$ & 0.0850 & 0.4309 & 0.0219 \\
\hline
\end{tabular}

Guaranteed minimum income benefits

For the GMIB, similarly to the GAO, the guaranteed annuity payment rate is set as

$$
g_{x_{0}, T}^{\mathrm{GMIB}}=\frac{T_{x_{0}}(0, T)}{T \mid \ddot{a}_{x_{0}}(0)},
$$

that is the GMIB is also "at the money". Since a closed-form solution for the value $V_{0}^{\mathrm{GMIB}}$ is not available in this case, we have to rely on simulations under the riskneutral measure $\mathbb{Q}$.

With respect to financial/economic risk, in contrast to the "Implementation" section from Application I where only simulations of $S_{1}$ and $r_{1}$ are necessary, here we additionally need to simulate the discount factor $\exp \left\{-\int_{0}^{T} r_{s} d s\right\}$. This can be achieved by sampling $r_{T}, \log \left\{A_{T}\right\}$, and $\int_{0}^{T} r_{s} d s$ simultaneously from their joint Normal distribution under $\mathbb{Q}$. We refer to Zaglauer and Bauer ${ }^{47}$ for details.

With respect to future mortality risk, we need simulations of $e^{-\int_{0}^{T} \mu_{s}\left(0, x_{0}+s\right) d s}$ and ${ }_{k-T} p_{x_{0}+T}(T, k), k=T, \ldots, \infty$, which analogously to the "Implementation" section from Application I can be expressed as

$$
\begin{aligned}
{ }_{T} p_{x_{0}}(T, T)= & e^{-\int_{0}^{T} \mu_{s}\left(0, x_{0}+s\right) d s}={ }_{T} p_{x_{0}}(0, T) \\
& \times \exp \left\{-\int_{0}^{T} \int_{0}^{s} \alpha\left(s-u, x_{0}+u\right) d u d s-\int_{0}^{T} C\left(x_{0}+s\right) Z_{s} d s\right\}, \\
k-T p_{x_{0}+T}(T, k)= & \frac{k p_{x_{0}}(0, k)}{T p_{x_{0}}(0, T)} \times \exp \left\{-\int_{0}^{T} \int_{0}^{k-T} \alpha\left(v+T-s, x_{0}+s\right) d v d s\right. \\
& \left.-\int_{0}^{k-T} C\left(x_{0}+T+v\right) e^{M v} d v Z_{T}\right\} .
\end{aligned}
$$

${ }^{47}$ Zaglauer and Bauer (2008). 
Table 7 Fair option fee $\phi^{*}$

\begin{tabular}{lcc}
\hline & Deterministic mortality & Stochastic mortality \\
\hline$\left(x_{0}, T\right)=(30,35)$ & 0.0278 & 0.0319 \\
$\left(x_{0}, T\right)=(40,25)$ & 0.0321 & 0.0349 \\
$\left(x_{0}, T\right)=(50,15)$ & 0.0423 & 0.0445 \\
\hline
\end{tabular}

Just as in the "Implementation" section from Application I above, the latter equations can be conveniently used in Monte Carlo simulations.

With the above simulation procedures, we can calculate the contract value $V_{0}^{\mathrm{GMIB}}\left(x_{0}, T\right)$ for given $\phi$. In particular, we are interested in the fair fee, that is the continuously deducted option fee $\phi^{*}$ such that $V_{0}^{\mathrm{GMIB}}=A_{0}$. Here we use the same age/ term combinations as in the previous section. The results are shown in Table 7.

We find that $\phi^{*}$ is greater when taking mortality risk into account, which appears intuitive since additional volatility naturally implies a relatively higher option price. However, we observe that the increase of $\phi^{*}$ is relatively modest in comparison to the results for the GAOs. Thus, it seems that while the effect of mortality risk is considerable relative to interest rate risk as shown in our analysis of GAOs, the influence is moderate relative to equity risk - the main risk driver for the VA/GMIB. In particular, our analyses show that mortality risk affects different mortalitycontingent options dissimilarly.

\section{Conclusion}

Due to their tractability and their compatibility with classical actuarial theory, Forward Mortality Factor Models present a convenient way of introducing systematic mortality risk to actuarial practice. This not only improves the accuracy of common actuarial calculations, but also helps to provide a more coherent "risk picture" of a life insurance company's operations.

The current paper documents the advantages of this model class by discussing in detail important example applications. In the first application, we derive the economic capital for a stylised life insurance company based on different monetary risk measures. Our results suggest that mortality risk has a considerable effect on the solvency of the company and thus should not be disregarded when assessing its financial stability. In the second application, we discuss the valuation of mortalitycontingent options, specifically GAOs and GMIBs within Variable Annuity contracts. Our analyses indicate that mortality risk affects the two options differently: While for simple GAOs, the values increase greatly with the inclusion of mortality risk, for the fair option fees for Variable Annuity contracts with GMIBs market risks dominate. However, it is important to note that the latter risk - at least to a large extent - is hedgeable whereas markets for mortality risk are only slowly maturing. Thus, while the influence of mortality risk on pricing may be relatively moderate in this case, the same may not necessarily be true for the associated profit and loss distribution. 
Moreover, here we assume a "financially optimal" exercise behaviour, which may not be true in practice; in particular, it is conceivable that mortality risk has an impact on the exercise behaviour.

\section{Acknowledgements}

We are thankful for helpful comments from conference participants and two anonymous referees. All remaining errors are ours. D. Bauer gratefully acknowledges financial support from the Willis Research Network.

\section{References}

Artzner, P., Delbaen, F., Eber, J-M. and Heath, D. (1999) 'Coherent measures of risk', Mathematical Finance 9(3): 203-228.

Ballotta, L. and Haberman, S. (2003) 'Valuation of guaranteed annuity conversion options', Insurance: Mathematics and Economics 33(1): 87-108.

Ballotta, L. and Haberman, S. (2006) 'The fair valuation problem of guaranteed annuity options: The stochastic mortality environment case', Insurance: Mathematics and Economics 38(1): 195-214.

Bauer, D. (2009) Stochastic Mortality Modeling and Securitization of Mortality Risk, Germany: ifa-Verlag, Ulm.

Bauer, D., Benth, F.E. and Kiesel, R. (2010a) Modeling the forward surface of mortality, Working Paper, Georgia State University.

Bauer, D., Bergmann, D. and Reuss, A. (2010b) On the calculation of the solvency capital requirement based on nested simulations, Working Paper, Georgia State University.

Bauer, D., Börger, M. and Ruß, J. (2010c) 'On the pricing of longevity-linked securities', Insurance: Mathematics and Economics 46(1): 139-149.

Bauer, D., Börger, M., Ruß, J. and Zwiesler, H.J. (2008a) 'The volatility of mortality', Asia-Pacific Journal of Risk and Insurance 3(1): 172-199.

Bauer, D., Kling, A. and Ruß, J. (2008b) 'A universal pricing framework for guaranteed minimum benefits in variable annuities', ASTIN Bulletin 38(2): 621-651.

Biffis, E. and Millossovich, P. (2006) 'The fair value of guaranteed annuity options', Scandinavian Actuarial Journal 2006(1): 23-41.

Björk, T. (1999) Arbitrage Theory in Continuous Time, Oxford: Oxford Finance.

Björk, T. and Gombani, A. (1999) 'Minimal realizations of interest rate models', Finance and Stochastics 3(4): 413-432.

Booth, H., Maindonald, J. and Smith, L. (2002) 'Applying Lee-Carter under conditions of variable mortality decline', Population Studies 56(3): 325-336.

Börger, M. (2010) 'Deterministic shock vs. stochastic value-at-risk-An analysis of the Solvency II standard model approach to longevity risk', Blätter DGVFM 31(2): 225-259.

Boyle, P.P. and Hardy, M.R. (2003) 'Guaranteed annuity options', ASTIN Bulletin 33(2): 125-152.

Brace, A., Gatarek, D. and Musiela, M. (1997) 'The market model of interest rate dynamics', Mathematical Finance 7(2): 127-155.

Cairns, A.J.G. (2007) A Multifactor Generalisation of the Olivier-Smith Model for Stochastic Mortality, proceedings of the $1^{\text {st }}$ IAA Life Colloquium, Stockholm.

Cairns, A.J.G., Blake, D. and Dowd, K. (2006) 'Pricing death: Frameworks for the valuation and securitization of mortality risk', ASTIN Bulletin 36(1): 79-120.

Cox, S.H. and Lin, Y. (2007) 'Natural hedging of life and annuity mortality risks', North American Actuarial Journal 11(3): 1-15.

Dawson, P., Dowd, K., Cairns, A.J.G. and Blake, D. (2010) 'Survivor derivatives: A consistent pricing framework', The Journal of Risk and Insurance 77(3): 579-596.

Girard, L.N. (2002) 'An approach to fair valuation of insurance liabilities using the firm's cost of capital', North American Actuarial Journal 6: 18-41. 
Glasserman, P. (2004) Monte Carlo Methods in Financial Engineering, New York: Springer.

Jamshidian, F. (1989) 'An exact bond option formula', Journal of Finance 44(1): 205-209.

Kalkbrener, M. (2005) 'An axiomatic approach to capital allocation', Mathematical Finance 15(3): 425-437.

Lee, R.D. and Carter, L.R. (1992) 'Modeling and forecasting U.S. mortality', Journal of the American Statistical Association 87(419): 659-671.

Milevsky, M.A. and Promislow, S.D. (2001) 'Mortality derivatives and the option to annuitize', Insurance: Mathematics and Economics 29: 299-318.

Pelsser, A. (2003) 'Pricing and hedging guaranteed annuity options via static option replication', Insurance: Mathematics and Economics 33: 283-296.

Schrager, D.F. (2006) 'Affine stochastic mortality', Insurance: Mathematics and Economics 38: 81-97.

Stevens, R., De Waegenaere, A. and Melenberg, B. (2010) Calculating capital requirements for longevity risk in life insurance products: Using an internal model in line with Solvency II, Working Paper, presented at the Sixth International Longevity Risk and Capital Markets Solutions Conference.

Zaglauer, K. and Bauer, D. (2008) 'Risk-neutral valuation of participating life insurance contracts in a stochastic interest rate environment', Insurance: Mathematics and Economics 43: 29-40.

Zhu, N. and Bauer, D. (2011) Coherent modeling of the risk in mortality projections: A semi-parametric approach, Working Paper, Georgia State University.

\section{About the Authors}

Nan Zhu received his BS and MS in the Department of Financial Mathematics at Peking University, and is currently a $\mathrm{PhD}$ candidate in the Department of Risk Management and Insurance at Georgia State University expected to graduate in the Summer of 2012. His research interests include stochastic mortality modeling and forecasting, applications of contract theory in insurance, financial mathematics, and the economics of life settlements.

Daniel Bauer is an Assistant Professor of Risk Management and Insurance in the Robinson College of Business at Georgia State University. He specialises in the development of new mathematical models for the valuation and risk management of insurance products and insurance-linked securities. Other research interests include insurance economics and mathematical finance. 


\section{Appendix}

\section{A principle component analysis}

In this appendix, we briefly outline the approach introduced in Zhu and Bauer ${ }^{5}$ for the estimation of the forward volatility function using principle component analysis.

Assume we are given a time series of generational mortality data $\left({ }_{\tau} p_{x}\left(t_{j}, t_{j}+\tau\right)\right)_{(\tau, x) \in \mathcal{C}}, j=1, \ldots, N, t_{j+1}-t_{j}=\Delta$, where $\mathcal{C}$ denotes a (large) collection of term/age combinations, $|\mathcal{C}|=K^{48}$ For each $(\tau, x) \in \mathcal{C}, j \in\{1,2, \ldots, N-1\}$, define

$$
\begin{aligned}
& F(j, \tau, x)=-\log \left\{\frac{\frac{\tau+1}{\tau+1+\Delta} p_{x-\Delta}\left(t_{j+1}, t_{j+1}+\tau+1\right)}{\left.\frac{\tau p_{x}\left(t_{j+1}+\tau+1\right)}{\tau+\Delta} t_{j+1}+\tau\right)}\right\} \\
& =-\log \left\{\frac{\frac{\tau+1}{{ }_{\tau} p_{x}\left(t_{j+1}, t_{j+1}+\tau+1\right)}}{\frac{\tau+1+\Delta p_{j+\Delta}\left(t_{j+1}, t_{j+1}+\tau+1\right)}{\tau+\Delta} p_{x-\Delta}\left(t_{j}, t_{j+1}+\tau\right)}\right\} \text {. }
\end{aligned}
$$

Intuitively, $F(j, \tau, x)$ measures the shift (log change) of the one-year marginal survival probability for the period $\left[t_{j+1}+\tau, t_{j+1}+\tau+1\right)$ from projection at time $t_{j+1}$ relative to time $t_{j}$, for an-at time $t_{j+1}-x$-year old. With some basic manipulations, we obtain

$$
\begin{aligned}
F(j, \tau, x) \stackrel{d}{=} & \int_{0}^{\Delta} \int_{\tau}^{\tau+1} \alpha(v+\Delta-s, x-\Delta+s) d v d s \\
& +\int_{0}^{\Delta} \int_{\tau}^{\tau+1} C(x+v) \exp \{M(v+\Delta-s)\} N d v d W_{s} \\
& =\int_{0}^{\Delta} \int_{\tau}^{\tau+1} \alpha(v+\Delta-s, x-\Delta+s) d v d s \\
& +\int_{\tau}^{\tau+1} C(x+v) \exp \{M v\} d v \times \int_{0}^{\Delta} \exp \{M(\Delta-s)\} N d W_{s}
\end{aligned}
$$

is Normal distributed. As is usual within a principle component analysis, we then

${ }^{48}$ For our dataset described in the section "Forward Mortality Factor Models", we have $t_{1}=1977, \Delta=1$, $N=31$, and $K=2,850$ since we rely on term/age combinations with $0 \leqslant \tau \leqslant 75,20 \leqslant x \leqslant 95$, and $20 \leqslant x+\tau \leqslant 95$. 
compute the empirical covariance matrix $\hat{\Sigma}$ of the i.i.d. vectors $\bar{F}_{j}=(F(j, \tau, x))_{(\tau, x) \in \mathcal{C}}$ and decompose it as

$$
\hat{\Sigma}=U \times\left(\begin{array}{cccc}
\lambda_{1} & 0 & \cdots & 0 \\
0 & \lambda_{2} & & 0 \\
\vdots & & \ddots & \vdots \\
0 & 0 & \cdots & \lambda_{K}
\end{array}\right) \times U^{\prime}=\sum_{v=1}^{K} \lambda_{v} u_{v} u_{v}^{\prime}
$$

where $U=\left(u_{1}, u_{2}, \cdots, u_{K}\right)$ is an (orthogonal) matrix consisting of the eigenvectors of $\hat{\Sigma}$ and $\lambda_{v}, v=1,2, \ldots, K$, are the corresponding (ordered) eigenvalues. Picking the $d$ greatest eigenvalues that explain the majority of the data, we obtain the approximation

$$
\bar{F}_{j} \approx \mathbb{E}\left[\bar{F}_{j}\right]+\sum_{v=1}^{d} u_{v} \sqrt{\lambda_{v}} Z_{v, j},
$$

where $Z_{v, j}$ are i.i.d. (scalar) standard Normal random variables, $1 \leqslant j \leqslant N-1$. In particular, when choosing $d=1$, we obtain

$$
\bar{F}_{j} \approx \mathbb{E}\left[\bar{F}_{j}\right]+u_{1} \sqrt{\lambda_{1}} Z_{1, j}
$$

and by evaluating or approximating the integrals in Eq. (13) we can find suitable candidates for the components $C(x), M$, and $N$-and thus for the volatility function-from the eigenvectors. For instance, when relying on a crude midpoint approximation for the first integral and an Euler approximation of the stochastic integral, we have

$$
u_{1} \sqrt{\lambda_{1}} \approx(\underbrace{C\left(x+\tau+\frac{1}{2}\right) \times \exp \left\{M\left(\tau+\frac{1}{2}+\Delta\right)\right\} \times N}_{\sigma\left(\tau+\frac{1}{2}+\Delta, x-\Delta\right)})_{(\tau, x) \in \mathcal{C}} .
$$

For the (re)estimation of parametric models that are developed based on this procedure, we can now take advantage of the known distribution of the vectors $\bar{F}_{j}$. In particular, we can explicitly take the drift condition (2) into account. We refer to Zhu and Bauer ${ }^{5}$ for more details. 